\title{
Ongoing Exposure of U.K. Infants to Vaccine Serotype Pneumococci
}

\section{To the Editors:}

We read with interest the article by Makwana et al.1 The authors note that most childhood invasive pneumococcal disease (IPD) cases are now due to non-13 valent pneumococcal conjugate vaccine (PCV13) serotypes though PCV13 serotypes remain in circulation with serotypes 3 and 19A listed among the top 10 that caused IPD in children in 2015/16. Of the 25 cases with PCV13-type IPD in 2015/16, 11 (44\%) were due to serotype $19 \mathrm{~A}$.

Key insight is given into the IPD burden in infants 3-11months of age, which over the 6 -year study period accounted for a substantial proportion (508/1255, 40\%) of the total in children $<5$ years of age. Additionally, meningitis was most prevalent in infants (209/456 cases, $45.8 \%$ ) and was associated with the highest case fatality (9.6\%). The most recent data from 2015/16 showed 103/271 (38\%) of IPD cases occurred in infants 3-11 months of age, with $5(4.8 \%)$ attributed to PCV13 serotypes. This reflects an increase in infant IPD compared with 2014/15 and slightly exceeds the number of cases in this age group in 2010/11 when PCV13 was first introduced illustrating a continuing and substantial burden of infant IPD.

In view of these findings, it is surprising that their implications are not discussed in the context of the U.K. proposal to remove a priming dose of PCV13 in infancy and introduce a reduced $1+1$ schedule. 2 Goldblatt et al. 3 found that the immunogenicity of a single dose of PCV13 at age 3 months was significantly lower than 2 doses at age 2 and 4 months, providing less direct protection in the months before the booster dose. Maternal immunization with a combined tetanus, diphtheria, acellular pertussis, inactivated polio vaccine (TdaP/IPV) also resulted in reduced postprimary geometric mean concentrations in infants in the $1+1$ group, but there was no effect in infants receiving 2 primary doses. 3 Since $\sim 74 \%$ of pregnant women received TdaP/IPV in 2017,4 any reduction in the postprimary response to a single PCV13 priming dose will further increase the vulnerability of many infants to IPD.

The basis for the $1+1$ proposal is that individual protection is less important in highincome countries with mature PCV programs because of substantially reduced carriage and transmission of vaccine serotypes. However, Makwana et al. clearly demonstrate that U.K. infants are frequently exposed to pneumococci with infant vaccine-type IPD persisting. This suggests removal of an infant priming dose at present risks leading to an increase in vaccinetype IPD, other pneumococcal syndromes and deaths in this most vulnerable age group, which is also supported by recent modeling.5 Furthermore, the U.K.'s experience with meningococcal $C$ vaccine provides a cautionary example of the danger of allowing financial considerations to take precedence over recommended schedules founded on large-scale clinical trials and years of postlicensure data. Infant cases of meningococcal $\mathrm{C}$ disease have increased following the removal of the infant meningococcal $C$ dose from the routine vaccination schedule in July 20166 with 1 infant left with particularly severe sequelae. We 
believe that Makwana's data indicate a similar increase in infant PCV13-type IPD will occur if the United Kingdom moves to a $1+1$ schedule.

Mary Slack, FRCPATH School of Medicine Griffith University, Gold Coast Campus Qld 4222, Australia

Andrew Vyse, PhD Harish Madhava, MD Pfizer Ltd Walton Oaks, United Kingdom

Ralf-Rene Reinert, MD Pfizer Inc Paris, France

Gillian Ellsbury, MD Carole Czudek, PhD Pfizer Ltd Walton Oaks, United Kingdom

Bradford Gessner, MD Pfizer Vaccines Collegeville, PA

\section{REFERENCES}

1. Makwana A, Shepard C, Borrow R, et al. Characteristics of Children With Invasive Pneumococcal Disease After the Introduction of the 13-valent Pneumococcal Conjugate Vaccine in England and Wales, 2010-2016. Pediatr Infect Dis. 2018:37:697703

2. Joint Committee on Vaccination and Immunisation. Draft Minute of the meeting on 04 October 2017. 2017; Available from: https:// app.box.com/s/iddfb4ppwkmtjusir2tc. Accessed June 20, 2018

3. Goldblatt D, Southern J, Andrews NJ, et al. Pneumococcal conjugate vaccine 13 delivered as one primary and one booster dose $(1+1)$ compared with two primary doses and a booster $(2+1)$ in UK infants: a multicentre, parallel group randomised controlled trial. Lancet Infect Dis. 2018;18:171-179

4. https://assets.publishing.service.gov.uk/ government/uploads/system/uploads/attachment_data/file/703479/hpr1518_prn tl- prtsssVC.pdf

5. Wasserman $\mathrm{M}$, Lucas $\mathrm{A}$, Jones $\mathrm{D}$, et al. Dynamic transmission modelling to address infant pneu- mococcal conjugate vaccine schedule modifica- tions in The United Kingdom. Epidemiol Infect. 2018;146:1797-1806

6. https://assets.publishing.service.gov.uk/government/uploads/system/uploads/attachment_data/ file/664858/Table_4_invasive_meningococcal_c_ infections_lab_reports_England_by_agegroup_epiyear.pdf 